\title{
Reliability of the Deep Abdominal Muscle Thickness Measurements Using Ultrasonography in Normal Subjects
}

\author{
YOSHITAKA OTANI, RPT ${ }^{1)}$, KEISUKE ITOTANI, RPT ${ }^{1)}$, \\ NORIAKI MAEDA, RPT, PhD ${ }^{1)}$, MiCHI IKADA, MT ${ }^{2}$, \\ MEgumi HatANAKA, MT ${ }^{2)}$, JUNICHI KATO, MD, $\mathrm{PhD}^{3)}$ \\ 1) Division of Physical Therapy, Hyogo Rehabilitation Center at Nishi-Harima: 1-7-1 Kouto, Shingu- \\ cho, Tatsuno city, Hyogo, 679-5165, Japan. TEL: +81 791-58-1050 \\ ${ }^{2)}$ Department of Clinical Laboratory \\ ${ }^{3)}$ Department of Internal Medicine
}

\begin{abstract}
Purpose] This study was performed to investigate the changes of thickness and reliability of the measurements of lateral abdominal muscles during respiratory maneuvers using ultrasonography. [Subjects and Methods] Participants were 10 healthy male volunteers (26.4 \pm 2.7 years old). Ultrasound B-mode scanning was performed with the subjects lying supine and the probe was placed transversely midway between the costal inferior border and the crista iliaca. The muscle thickness of the external oblique (EO), internal oblique (IO), and transverses abdominal (TA) were measured under two conditions: resting expiration (Rex) and maximum expiratory effort (MEE). Percent thickness change was calculated as thickness ${ }_{\mathrm{MME}}-$ thickness $_{\mathrm{Rex}} /$ thickness $_{\mathrm{Rex}} \times 100 \%$. Interclass correlation coefficients (ICC 1,2) were used to estimate reliability. [Results] Muscle thickness during MME were significantly increased compared with that during Rex and the EO thickness was significantly decreased. Intraexaminer reliability estimates ranged from 0.97 to 0.82 for $\mathrm{EO}, 0.94$ to 0.81 for IO and 0.67 to 0.86 for TA. [Conclusions] We conclude that the thickness of the lateral abdominal muscles is influenced by voluntary respiration, and the assessment of the thickness by means of ultrasonography is adequately reliable. This information may be useful for deciding exercise prescription.

Key words: Ultrasonography, Deep abdominal muscles, Reliability
\end{abstract}

(This article was submitted Oct. 5, 2010, and was accepted Nov. 11, 2010)

\section{INTRODUCTION}

Ultrasonography is capable of noninvasively evaluating the dynamic function of soft tissues, such as muscles, ligaments, and tendons, and is essential in sports rehabilitation ${ }^{1)}$. It has recently been reported that strengthening of the deep muscles of the trunk, evaluated by ultrasonography, stabilized the lumbar region in lumbago patients $^{2,3)}$.

The lateral abdominal muscle is known to be involved in various functions, such as trunk movement, intra-abdominal pressure elevation, and expiration. To investigate these functions, evaluation of not only the external (EO) and internal (IO) oblique muscles but also the transversus abdominis muscle (TA) is necessary. Previous studies on the evaluation of deep trunk muscles using ultrasonography showed the high level of reliability of measuring muscle thicknesses in ultrasonographic images ${ }^{4,5}$. However, additional investigation of thicknesses under different image capture conditions in a resting state and the reliability of measurements during voluntary contraction is necessary.

The objective of this study was to measure the lateral abdominal muscle thickness in healthy subjects in ultrasonographic images, identify changes in the thickness between resting respiration and maximal expiratory effort, and investigate the reliability of the findings.

\section{SUBJECTS AND METHODS}

The subjects were 10 healthy men (age: $26.4 \pm 2.7$ years) who gave their consent to participate in this study. The lateral abdominal muscle thickness was measured using a 3.5-MHz B-mode linear ultrasonograph (PHILIPS HD11XE, USA).

The subjects rested in a supine position on a bed. The measurement sites were the costal margin on the right anterior axillary line and the center of the iliac crest. The probe was placed so as to align its major axis perpendicular to the right anterior axillary line with its center on the line. One examiner positioned the transducer and optimized the 


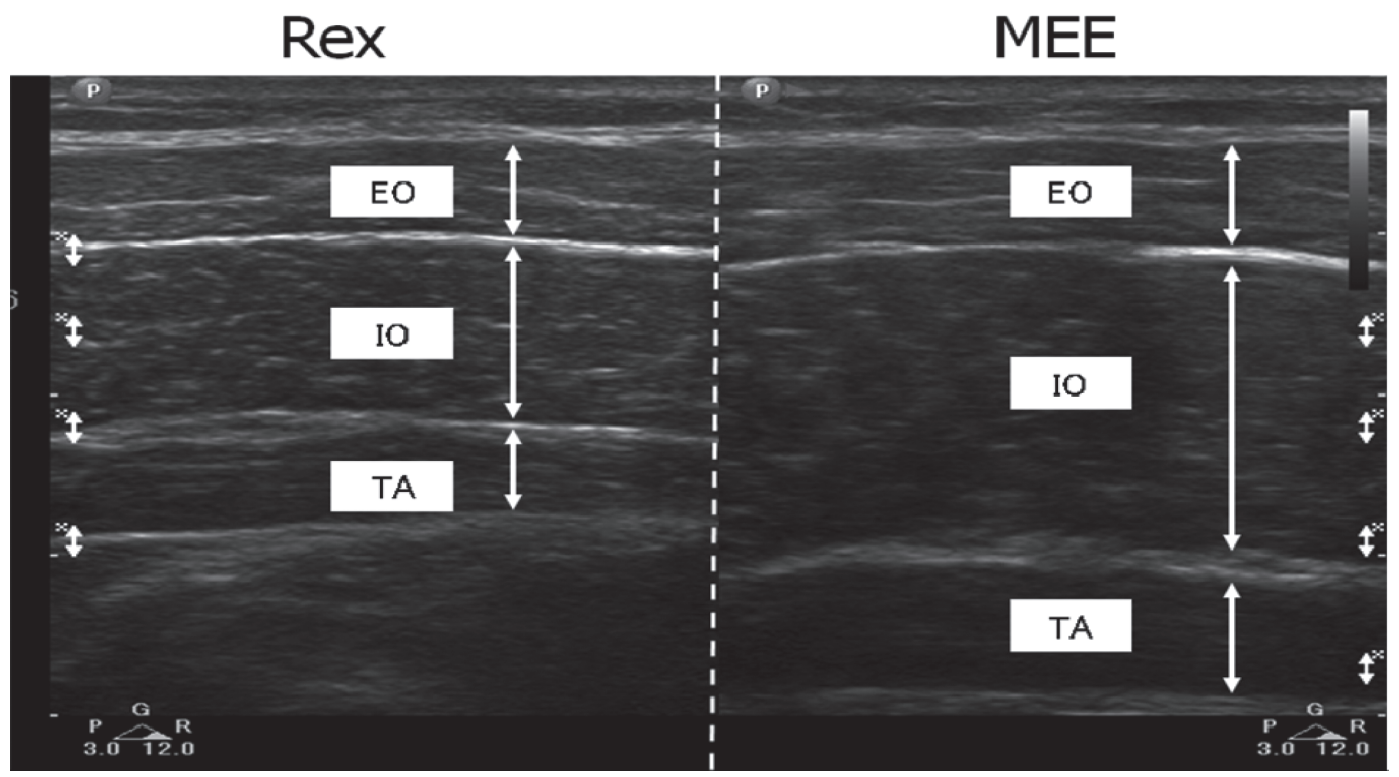

Fig. 1. Ultrasonography of the three muscles of the lateral wall of the abdomen at resting end expiration (Rex) and maximal expiratory effort (MEE)

EO : external oblique, IO : internal oblique, TA : transverses abdominis

quality of the image, and the same examiner captured and saved the image. The probe position and gain of the ultrasonography system were fine-adjusted to display the boundaries of EO, IO, and TA on the monitor. The lateral abdominal muscle thickness was measured under 2 conditions: resting end expiration (Rex) and maximal expiratory effort (MEE). In Rex, subjects were instructed "Please gently breathe out through your mouth". The instruction given for MEE was "Please use all of your strength to breath out through your mouth". During Rex and MEE at the end of expiration, the ultrasound image was frozen on the monitor and then the thickness of each muscle was measured. To compare the lateral muscle thicknesses (EO, IO, and TA) between the conditions (Rex and MEE), the means of 3 measurements were adopted as representative values of the muscles. The ultrasonographic images of the EO, IO, and TA thicknesses at Rex and MEE are shown in Figure 1.

The rate of change in muscle thickness (\%) was calculated employing the following equation: (MEE muscle thickness-Rex muscle thickness / Rex muscle thickness) $\times$ $100(\%)$. Differences in muscle thickness were compared employing the Kruskal-Wallis test, and values less than 5\% were considered significant.

The intra-class correlation coefficient (ICC 1,2) was also calculated to investigate the within-subject reliability of each muscle thickness.

\section{RESULTS}

The lateral abdominal muscle thicknesses (EO, IO, and TA) under respiratory conditions of Rex and MEE and the rate of change in each muscle are shown in Table 1. Compared to the thickness at Rex, the EO thickness was significantly decreased at MEE, from $0.60 \pm 0.17$ to $0.52 \pm 0.11 \mathrm{~cm}(\mathrm{p}<0.05)$, whereas those of IO and TA were significantly increased from $0.91 \pm 0.14$ to $1.28 \pm 0.26$ and from $0.31 \pm 0.04$ to $0.63 \pm 0.14 \mathrm{~cm}$, respectively $(\mathrm{p}<0.01)$. The rates of change in EO, IO, TA thicknesses were $-11.0 \pm 12.1,41.3 \pm 16.8$, and $100.0 \pm 33.8 \%$, respectively.

The ICC values $(1,2)$ are shown in Table 2 . The values of EO ( $\mathrm{ICC}=0.97)$ and $\mathrm{IO}(\mathrm{ICC}=0.94)$ at Rex showed a high level of reliability of the thickness measurement, but that of TA (ICC $=0.67$ ) was low. At MEE, the ICC values of all muscles: $\mathrm{EO}(\mathrm{ICC}=0.82)$, IO $(\mathrm{ICC}=0.81)$, and TA (ICC $=0.86)$, showed marked reliability.

Table 1. Mean muscle thickness at resting end expiration (Rex) and maximal expiratory effort (MEE) $(\mathrm{n}=10)$

\begin{tabular}{lccc}
\hline & Rex $(\mathrm{cm})$ & MEE $(\mathrm{cm})$ & $\%$ change \\
\hline EO & $0.60 \pm 0.17$ & $0.52 \pm 0.11^{*}$ & $-11.0 \pm 121$ \\
IO & $0.91 \pm 0.14$ & $1.28 \pm 0.26^{* *}$ & $41.3 \pm 16.8$ \\
TA & $0.31 \pm 0.04$ & $0.63 \pm 0.14^{* *}$ & $100.0 \pm 33.8$ \\
\hline & & & $* \mathrm{p}<0.05, * * \mathrm{p}<0.01$
\end{tabular}

Table 2. Interexaminer reliability using the means of 3 measures at resting end expiration (Rex) and maximal expiratory effort (MEE) $(n=10)$

\begin{tabular}{ccc}
\hline & Rex & MEE \\
\hline EO & 0.97 & 0.82 \\
IO & 0.94 & 0.81 \\
TA & 0.67 & 0.86 \\
\hline
\end{tabular}




\section{DISCUSSION}

Using ultrasonography, the lateral abdominal muscle thickness was measured in resting expiration and at MEE, and changes in the muscle thicknesses and their measurement reliabilities were investigated. Compared to with their values at Rex, the IO and TA thicknesses significantly increased at MEE, and the rate of change in the TA thickness was the greatest. The lateral abdominal muscle becomes readily mobilized as the posture changes from recumbence to the seated and standing positions ${ }^{6,7)}$.

Kaneko et al. reported that the thickness of the lateral abdominal muscles (EO, IO, and TA) increased at MEE, and the rates of change were 20,60 , and $86 \%$, respectively ${ }^{8}$. In our study, the rates of change were -11 , 41 , and $100 \%$, respectively, showing notable difference. One reason for the differences may have been the supine position adopted for measurement, which may have less influence on the intra-abdominal pressure at MEE compared to the seated and standing positions.

Regarding the reliability of measurement using ultrasonography, Misuri et al. ${ }^{9)}$ measured the lateral abdominal muscle thickness during resting respiration in healthy subjects and observed a low variation coefficient for each muscle thickness, but their measurements at MEE were repeated until the variation of 3 measurements decreased to less than $5 \%$, and they did not discuss the measurement reliability. In our study, a high $\operatorname{ICC}(1,2)$ (0.81-0.86) was obtained at MEE, suggesting that ultrasonographic measurement at MEE may be a useful means of evaluating lateral abdominal muscle function.

It has recently been reported that strengthening of the deep muscles of the trunk, evaluated by ultrasonography, stabilized the lumbar region in lumbago patients ${ }^{10,11)}$.

Hodges et al. ${ }^{12)}$ measured the lateral abdominal muscle thickness at maximum voluntary contraction, and observed that measurement was difficult due to morphological changes in some subjects. Measurement of morphological changes in the lateral abdominal muscle at MEE by muscle thickness measurement is limited because the measurement is one-dimensional. To identify changes in individual muscles, it is necessary to increase the reliability of muscle thickness changes by employing 3-dimensional measurements, such as those which can be obtained using MRI, and this is a research subject which remains to be investigated.

\section{REFERENCES}

1) Hides JA, Richardson CA: Use of real-time ultrasound imaging for feedback in rehabilitation. Manual Therapy, 1998, 3: 125-131.

2) Critchley DJ, Coutts FJ: Abdominal muscle function in chronic low back pain patients. Measurement with real-time ultrasound scanning. Physiotherapy, 2002, 88: 322-332.

3) Ferreira PH, Ferreira ML, Hodges PW: Changes in recruitment of the abdominal muscles in people with low back pain. Ultrasound measurement of muscle activity. Spine, 2004, 29: 2560-2566.

4) Kaneko H, Sato H, Maruyama H: Reliability of lateral abdominal muscles thickness measurement using ultrasonography. J Phy Ther Sci, 2005, 20: 197-201.

5) Koppenhaver SL, Hebert JJ, Fritz JM, et al.: Reliability of rehabilitative ultrasound imaging of the transversus abdominis and lumbar multifidus muscles. Arch Phys Med Rehabil, 2009, 90: 87-94.

6) De Troyer A: Mechanical role of abdominal muscles in relation to posture. Respir Physiol, 1982, 53: 341-351.

7) Snijders CJ, Bakker MP, Vleeming A, et al.: Oblique abdominal muscle activity in standing and in sitting on hard and soft seats. Clin Biomech, 1995, 10: 73-78.

8) Kaneko H, Sato H, Maruyama H : Influence of posture on lateral abdominal muscles' thickness. Phy Ther Sci, 2006, 21: 255-259.

9) Misuri G, Colagrande M, Gorini I, et al.: In vivo ultrasound assessment of respiratory function of abdominal muscles in normal subjects. J Eur Respir J, 1997, 10: 2861-2867.

10) Endleman I, Critchley DJ: Transversus abdominis and obliquus internus activity during pilates exercise: Measurement with ultrasound scannning. Arch Phys Med Rehabil, 2008, 89: 2205-2212.

11) Teyhen DS, Rieger J, Westrick RB, et al.: Changes in deep abdominal muscle thickness during common trunk-strengthening exercises using ultrasound imaging. J Orthop Sports Phys Ther, 2008, 38: 596-605.

12) Hodges PW, Pengel LHM, Herbert RD, et al.: Measurement of muscle contraction with ultrasound imaging. Muscle Nerve, 2003, 27: 682-692. 